\title{
A Window of Opportunity: Designing Carbon Nanomaterials for Environmental Safety and Health
}

\author{
Lin Guo ${ }^{1, a}$, Xinyuan Liu ${ }^{1, b}$, Vanesa Sanchez ${ }^{1, c}$, Charles Vaslet ${ }^{1, d}$, \\ Agnes B. Kane ${ }^{1, \mathrm{e}}$, and Robert H. Hurt ${ }^{1, \mathrm{f}}$ \\ ${ }^{1}$ Brown University, Providence, Rhode Island, 02912 USA \\ aLin_Guo@brown.edu, bXinyuan_Liu@brown.edu, Vanesa_Sanchez_Ld@brown.edu, \\ ${ }^{\mathrm{d}}$ Charles_Vaslet@brown.edu, ${ }^{\mathrm{e}} \overline{\mathrm{A}} \mathrm{gnes}$ KKane@brown.edu, ${ }^{\mathrm{f}}$ Robert_Hurt@brown.edu
}

Keywords: carbon nanotubes, fullerenes, toxicity, purification, surface chemistry

\begin{abstract}
Carbon nanomaterials are among the best known and most promising products of the nanotechnology movement. Some early studies suggest that fullerenes and nanotubes may pose significant health risks, and this has given rise to an emerging literature on carbon nanotoxicology. This young field has now begun to yield insight into toxicity mechanisms and the specific material features involved in those mechanisms. This paper explores the potential to alter those material features through post-processing or reformulation with the goal of reducing or eliminating carbon nanomaterial health risks. The paper emphasizes the important roles of metal content and bioavailability, carbon surface chemistry, and nanomaterial aggregation state. The nanotechnology movement has been given a unique "window of opportunity" to systematically investigate the toxicity of nanotechnology products and to develop ways to manage health risks before large scale manufacturing becomes widespread.
\end{abstract}

\section{Introduction}

The world-wide nanotechnology movement is devoting an increasing fraction of its development funding to the parallel investigation of potential health and environmental effects. In the last few years, conflicting data have emerged about the biocompatibility and toxicity of both fullerenes and carbon nanotubes [1,2]. An initial study in 2001 by Huczko et al. [3] concluded that "working with soot containing carbon nanotubes is unlikely to be associated with any health risk" after investigated the effects of CNT on the pulmonary function of guinea pigs. Lam et al. [4] studied the pulmonary toxicity of three batches of SWNTs and they believed that these SWNTs containing metal impurities "induced dose-dependent lung lesions characterized chiefly by interstitial granulomas". Cui et al. [5] showed that "SWNTs can inhibit HEK293 cell proliferation, decrease cell adhesive ability in a dose- and time-dependent manner". Meanwhile, there also have been a number of published studies that support the biocompatibility of CNT. Kam et al.[6] studied the uptake of SWNT-streptavidin conjugates into human cells via endocytosis and no cytotoxicity was observed for the pristine (unconjugated) SWNTs. Fullerenes were reported to induce oxidative stress in the brain of juvenile largemouth bass [7] and recent studies show that fullerene toxicity depends on surface functionalization [8] and may depend on the presence of residual solvent used to create soluble C60 clusters (so-called nano-C60) in aqueous solution $[9,10]$. To resolve these issues, further study is needed.

As the emerging field of nanotoxicology progresses, there will be increasing focus on the mechanistic basis of toxicity with the goal of understanding the contributions of specific material features, such as size, shape, surface chemistry, and metals content. As we learn more about how these features influence toxicity, we open up the possibility of modifying or reformulating the materials to reduce, or in some cases perhaps eliminate, their potential to harm the environment and human health. Here 
we consider how the principles of eco-materials design can be applied to new nanomaterials now, in the current "window of opportunity" before the products are manufactured and commercialized on a large scale.

\section{The Role of Catalytic Metals}

Of the various routes to carbon nanotubes, the catalytic routes have become dominant, and as a result almost all of the CNT samples produced commercially contain metals (see Table 1, Fig. 1). The most abundant CNT metals are Fe and Ni, but Y, Co, and Mo are also common. There is a significant literature on metals toxicology, and although it is not targeted at CNT exposures, it provides a useful starting point.

Table 1. Residual metals content in commercial carbon nanotube samples

SWNT samples
Vendor 1, as-produced: $\mathrm{Ni}-\mathrm{Y}(35 \%)$
Vendor 2, as-produced: $\mathrm{Ni}-\mathrm{Co}(25 \%)$
Vendor 3, as-produced: $\mathrm{Ni}-\mathrm{Y}(25 \%)$
Vendor 3, alternate process: Co-Mo $(<2 \%)$
Vendor 4, as-produced: $\mathrm{Ni}-\mathrm{Y}(30 \%)$; purified: Ni-Y $(15 \%)$
Vendor 5, as produced: $\mathrm{Fe}(22.2 \%)$; purified: Fe $(10.9 \%)$

MWNT samples

Vendor 6: as-produced: Fe (4.2\%) purified: $\mathrm{Fe}(0.1 \%)$

Vendor 7: as-produced: Fe $(4.2 \%)$ purified: $\mathrm{Fe}(3.3 \%)$

Nickel. There have been numerous studies of the toxicity of nickel and its compounds. Models of nickel-induced respiratory tumors suggest that the bioavailability of nickel (the delivery of $\mathrm{Ni}$ (II) ions to the nucleus of target epithelial cells) is the major determinant for the carcinogenicity of nickel [11-13]. A key material science question, therefore is whether sufficient $\mathrm{Ni}$ (II) ion can be released from CNT samples. Moreover, the ability of various nickel compounds to be taken up by cells directly influences intracellular nickel levels, and their carcinogenic activity is proportional to cellular uptake $[11,12]$. Soluble nickel ions can be transported directly and insoluble or relatively insoluble nickel compounds can be endocytosed or phagocytosed. Phagocytosis/endocytosis of poorly soluble nickel compounds such as $\mathrm{Ni}_{3} \mathrm{~S}_{2}$ and $\mathrm{NiO}$ may play an important role in the pulmonary toxicity and carcinogenicity of nickel. Direct delivery of nickel nanoparticles to the lungs of rodents induced acute injury and inflammation persisting up to one month [14].

Iron Iron is abundant in the earth's crust and is an essential element that is tightly regulated by specialized iron transport and storage proteins in the body. Free iron, however, has been hypothesized to contribute to the pulmonary toxicity of ambient ultrafine particles [15], asbestos [16] and most recently human keratinocytes exposed in culture to Fe-containing SWNTs [17]. The toxicity of amphibole asbestos fibers, which are fibrous silicates containing iron with a high aspect ratio and physiochemical properties similar to carbon nanofibers and nanotubes, is also hypothesized to be related to generation of reactive oxygen species [18-20]. In carbon nanotubes iron is in close association with carbon and is quenched from high-temperature reducing environments used for CNT growth. The high concentration of Fe in some samples (Table 1) coupled with its reduced state and nanoparticle form make Fe-induced toxicity in CNTs a concern that requires systematic investigation.

Y, Co, Mo Yittrium is the most common second element in bimetallic catalysts used in the arc process for SWNTs, a typical formulation being 4:1 Ni-Y. Yittrium is a rare-earth metal for which the toxicological database is more limited than for other CNT metals. Hirona et al. [21] studied the intratracheal instillation of $\mathrm{YCl}_{3}$ in rats and found that $\mathrm{Y}$ was localized in lysosomes of alveolar and interstitial macrophages and basement membranes. $\mathrm{YCl}_{3}$ instillation induces acute lung injury; however, it 
persists in the lungs after instillation or intravenous delivery [22,23]. Chronic or sustained release of Y from SWNTs may produce chronic lung or systemic toxicity. In contract, $\mathrm{Y}_{2} \mathrm{O}_{3}$ nanoparticles have been shown to protect nerve cells in culture from oxidant-induced injury [24]. Nanotube-derived yttrium has the potential to induce pulmonary toxicity, but this issue has not been investigated to our knowledge.

Cobalt is an essential trace element, but excess dietary cobalt is known to produce toxic effects in mammals [25]. Inhalation of cobalt is reported to induce asthma, and has been implicated in so-called "hard metal disease" in metal workers [26].

Together $\mathrm{W}$ and Co catalyze formation of reactive oxygen species that lead to oxidative stress and lung toxicity [26]. Epidemiology suggests a lower risk for exposure to cobalt alone [26]. Cobalt particles have been reported to be genotoxic in vitro in human peripheral blood mononucleated cells and were classified as possibly carcinogenic to humans by IARC in 2003 [27]. Cobalt(II) ions can be released from metallic cobalt particles under physiological conditions and have been reported to cause cell injury and lung tumors in rodents [27]. Intratracheal instillation of cobalt nanoparticles was less potent than nickel nanoparticles or quartz in inducing acute lung injury in rodents; however, cobalt nanoparticles were more toxic than $\mathrm{TiO} 2$ nanoparticles used in this study [28]. Molybdenum is an essential trace element for which inhalation toxicity data on humans is quite limited.
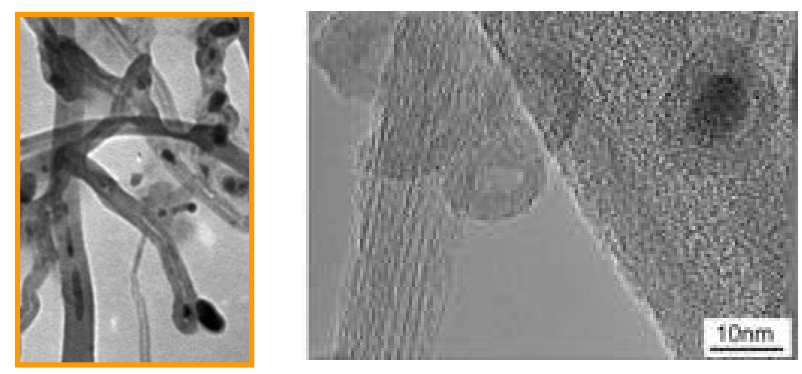

Figure 1. Morphologies of metal catalyst residues in carbon nanotubes. Left: iron nanoparticles and nanorods found inside and outside the central nanotube hollow core. Center: purified SWNT sample showing a nickel nanoparticle encased in carbon as well as empty carbon shells. The TEM image alone is not sufficient to reveal if carbon provides isolation from fluid phases.

Applying this toxicological information on pure metals and their compounds to CNT toxicology is a challenge for several reasons. First the CNT metals are nanoscale particles, which often exhibit higher biological activity compared to conventional micron-scale powers. Secondly, the metal phases in CNT samples are often poorly characterized and complex. Finally and most importantly, CNT metallic residues are at least partially encapsulated by carbon shells as seen in Fig. 1. Because the molecular mechanisms of metal toxicity usually involve soluble species (removed from particles by dissolution or chelation), these metal particles should only be active if they are accessible to the surrounding physiological fluids. Fluid accessibility or "bioavailability" is the single most important issue governing metals contributions to CNT toxicity. Our work has shown that TEM analysis is not sufficient to determine bioavailability, and Figure 2 show examples of specific assays we have developed to measure this important parameter.

\section{The Role of Carbon Surface Chemistry}

Recent evidence indicates that surface chemistry is an important factor in the toxicity of carbon nanomaterials. Carbon surfaces are often described as "hydrophobic" but in fact their surfaces vary greatly in hydrophobicity depending on the number and form of surface functional groups introduced by air or acids used in purification schemes. As-produced carbon nanomaterials have water contact angles from 40 - 90 degrees [29] and more recent data from our laboratories demonstrate treatment protocols that allow systematic variation of water contact angle over the entire range of 5 to 167 degrees [30]. 
Several studies have shown that surface functionalization to increase hydrophilicity (water solubility) tends to decrease the toxicity of carbon nanomaterials. Sayes et al. [8] report that hydroxylation of fullerenes reduces their toxicity by up to $10^{7}$ (ratio of doses for same percentage cell death). The authors observe oxidative damage to cell membranes in all cases where fullerene exposure led to cell death [8]. The lowered toxicity may be due to a decreased tendency of the hydrophilic materials to associate with cell membranes or to decreased redox activity as a result of hydroxylation. Dumortier et al. [31] show the nanotube toxicity is also sensitive to surface functionalization, and the degree of water solubility in particular. Functionalizing nanotubes by an oxidation/amidation treatment to introduce negatively charged surface groups produces water soluble CNTs that do not exert toxic effects on immune cells, B and T lymphocytes [31]. In contrast, the less-soluble formulation provokes secretion of proinflammatory cytokines from macrophages [31]. The authors point out that a variety of studies have now shown that soluble CNTs exhibit
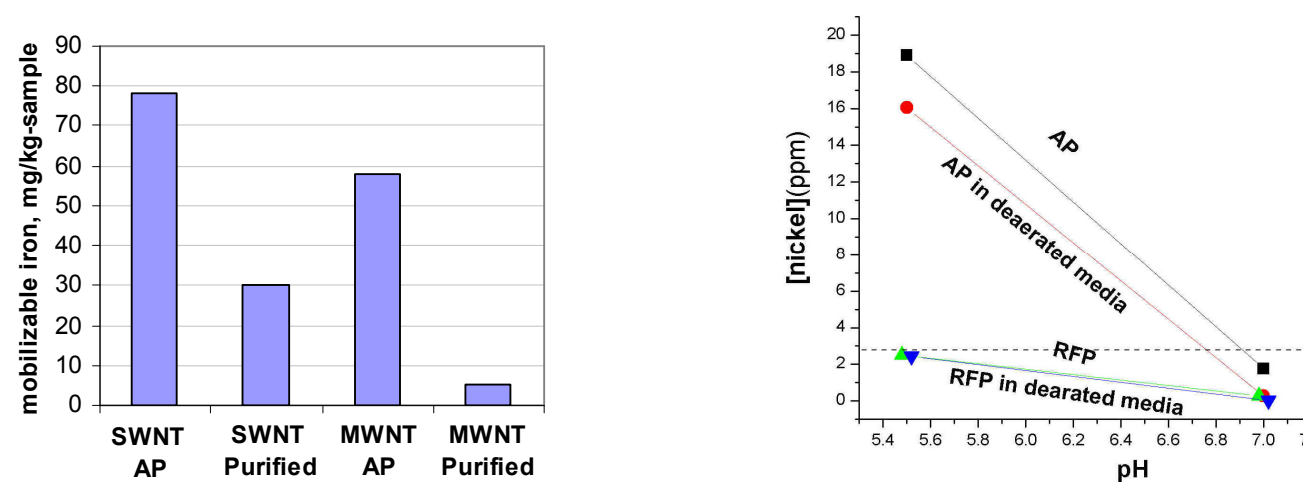

Figure 2. Measurements of free iron and nickel in carbon nanotube samples. Top: Fe mobilized from four commercial nanotube samples comprising two pairs of as-produced and purified forms. Purification reduces the free Fe, but does not eliminate it. The soluble iron is measured by colorimetric determination of ferrozine- $\mathrm{Fe}^{2+}$ complex and the solubility assay is performed for three days in the presence of the chelator, ferrozine, and the physiological reducant, ascorbate. Bottom: Ni mobilized from a commercial single-wall nanotube samples at both extracellular $\mathrm{pH}(7)$ and typical $\mathrm{pH}$ in cellular lysosomes (5.5). Nickel determined by ICP following $2 \mathrm{hr}$ incubation of $1 \mathrm{mg}-\mathrm{CNT} / \mathrm{ml}$-solution. The dashed line is the $\mathrm{LC}_{50}$ value for a prior study of nickel ion toxicity toward fibroblast cells (Tiara et al).

low toxicity toward several immortalized cell lines such as murine fibroblasts, and human promyelocytic leukemia cells. Overall, systematic surface modification is a promising approach for reducing the toxicity of some carbon nanomaterials.

\section{The Role of Aggregation State}

Two aspects of nanotoxicology that require more attention are the effects of carbon structure and aggregation state. The fibrous or tubular dimension is important as it influences the ability of macrophages, the cells responsible for clearing foreign objects from the lung, to phagocytose nanomaterials deposited in the lung and remove them from the airways by transport up the mucocilliary escalator. An approximate general rule is that fibers or nanofibers may be difficult to phagocytose if their lengths significantly exceed the diameter of the phagocytic cell $(10-20 \mu \mathrm{m})$. The phenomenon of "frustrated phagocytosis" is a characteristic of macrophage interaction with some asbestos fibers. Mechanisms of phagocytosis and frustrated phagocytosis of nanotubes requires more study and may be length dependent.

Human heath risks will depend on nanomaterial aggregation state. The hydrophobic nature of many carbon nanomaterials, coupled with the fibrous nature of CNTs that 
allow entanglement, give rise to aggregates both in solution and in the dry state. Aggregate sizes range from small multiples of the primary tube size $(\sim 100 \mathrm{~nm})$ to large aggregates (microns to millimeters) and interwoven networks of essentially unlimited (macroscopic) dimension. Aggregation or entanglement greatly decreases the likelihood of inhalation exposure by reducing the concentration of airbore material and also by reducing the fraction of that material in the respirable size range $(<10$ $\mu \mathrm{m})$. In addition, aggregation state may influence cytotoxicity by altering the physical interaction between target cells and nanomaterials. Figure 3 shows how the interaction of macrophages with carbon nanofibers changes with aggregation state. There is potential to minimize nanotube toxicity by casting and delivering them in entangled mats or polymer matrices that limit aerosol formation and cellular uptake.

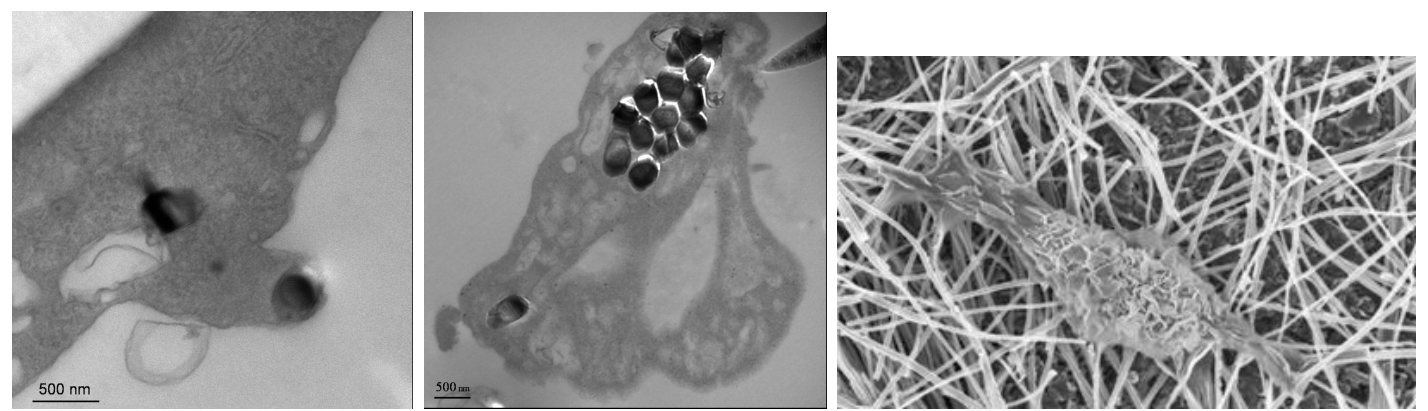

Figure 3: The effect of aggregation state on cell/nanomaterial interactions. Left: TEM thin sections showing single carbon nanofibers in the process of internalization into macrophages; Center: thin section showing a CNF bundle fully internalized by macrophages after 1 hour exposure; Right: SEM image of a macrophage adhered to a carbon nanofiber network. Such matting may prevent phagocytosis and instead promote cell attachment and spreading.

\section{Outlook: the potential for eco-nanomaterials}

Carbon nanomaterial samples are typically complex mixtures and the limited information available suggests that their toxicity depends on the specific formulation, in particular: (i) hydrophilicity as determined by surface functionalization, (ii) metals content and bioavailability, and (iii) state of aggregation which influences respirability as well as cell-material interactions. While these dependencies complicate toxicity testing, they also provide an opportunity for manufactures to formulate nanomaterials specifically to minimize health risk.

Figure 4 shows the potential of reformulation or post-treatment to reduce health risk in a variety of exposure scenarios. The common practice of supplying as-produced materials to laboratories and applications-oriented companies may increase health risks in each of the exposure scenarios shown. An alternative is to purify or postprocess (e.g. functionalize) for reduced toxicity at the point of manufacture. If this were common practice one could reduce the health risk associated with uncontrolled exposures 3-6 in the Figure. This option eliminates the shipping of as-produced material as depicted by the " $X$ " in the diagram. This option may be particularly attractive for mitigation of metals effects, since metals are rarely required for CNT applications and are usually undesirable impurities. Even if purification is done universally, one still needs engineering controls to limit exposure (respirators, filters, sealed reactors) for the pre-purification routes 1 and 2 in Fig. 4 . Understanding the contributing factors to nanotube toxicity should be a high priority effort during the current "window of opportunity" prior to large scale commercialization.

\section{Acknowledgements}

The authors acknowledge the technical contributions of Daniel Morris, David Murray, Joseph Orchardo, Paula Weston, Prof. Anatoly Zhitkovich, and Prof. Joseph Calo at 
Brown University. Financial support from the NIEHS (SBRP grant P42 ES013660), National Science Foundation (NIRT grant DMI-0506661), and the U.S. EPA (STAR grant 83171901) at Brown University is gratefully acknowledged.

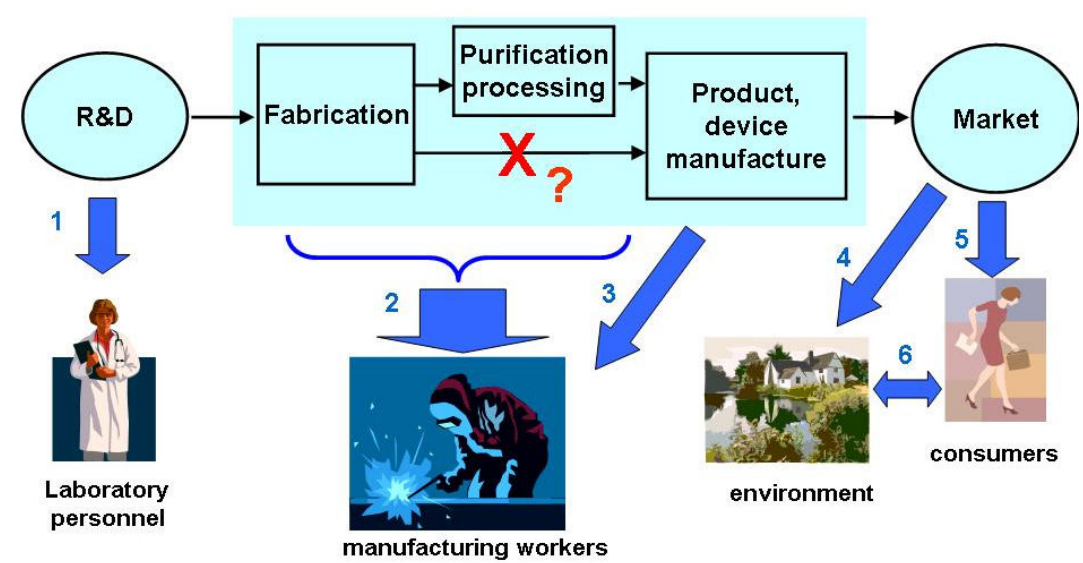

Figure 4. Exposure routes during various stages of the nanomaterial life cycle.

\section{References}

1. S.K. Smart, A.I., Cassady, G.Q. Lu, and D.J. Martin, Carbon 44 (2006) p. 1034

2. R. Hurt, M. Monthioux A. Kane, Carbon 200644 1028-1033

3. A. Huczko, H. Lange, E. Calko, H., Grubek-Jaworska, P. Droszcz, Full. Sci Tech 9 (2001), p. 251

4. C. Lam, J.T. James, R. McCluskey, R. Hunter, Toxicol Sci 77 (2004) p. 126

5. D. Cui, F. Tian, C.S. Ozkan, M., Wang, H., Gao, Toxicol Lett 155 (2005) p. 73

6. N.W.S. Kam, T.C. Jessop, P.A. Wender, and H. Dai, J Am Chem Soc 126, (2004) p. 6850.

7. E. Oberdörster, Environ Health Perspect, 112 (2004) p. 1058

8. C.M. Sayes, J.D. Fortner, W. Guo, D. Lyon, A.M. Boyd, K. D. Ausman, Y.J. Tao, B. Sitharaman,

L.J. Wilson, J.B. Hughes, J.L. West, V.L. Colvin, Nano Letters, 4 (2004) p. 1881.

9. J. L. Fortner, DY, C. M. Sayes, A. M. Boyd, J. Falkner, E. Hotze, L. Alemany, Y.

Tao, K. Ausman, V. Colvin and J. Hughes, Environ Sci. Technol 39 (2005) P. 4307

10. E. Oberdörster, S. Zhu, T M. Blickley, P. McClellan-Green, M.L. Haasch, Carbon 44 (2006) p1112.

11. A.R. Oller, M. Costa, G. Oberdorster, Tox. Appl. Pharmacol. 143 (1997) p. 152

12. M. Costa and H.H. Mollenhauer, Science, 209 (1980) p. 515

13. E.T. Snow and M. Costa, Environmental and Occupational medicine; Lippincott-Raven Publishers: Philadelphia Chapt 75 p. 1057 (1998).

14. Q. Zhang T. Kusaka, K. Sato Y. Mo, M. Fukuda, K. Donaldson, J. Occup. Health 40 (1998) 171.

15. F. Tao, B. Gonzalez-Flecha, L. Kobzik, Free Rad. Biol. \& Med. 35 (2003) p. 327

16. J.A. Hardy and A.E. Aust, Chem. Rev. 95 (1995) p. 97

17. A.A. Shvedova, et al. J. Toxicol. Environ. Health., Part A 66 (2003) p. 1909

18. K. Donaldson, R. Aitken, L. Tran, V. Stone, R. Duffin, G. Forrest, A. Alexander Toxicol. Sci. 91 (2006) p. 5.

19. B. Fubini and C.O. Areán, Chem. Soc. Rev. 28 (1999) p. 373.

20. A. Shukla, et al. Free Rad. Biol. \& Med. 34 (2003) p. 1117.

21. S. Hirano, N. Kodama, K. Shibata, K.T. Suzuki, Toxicol. Appl. Pharmacology, 104 (1990) p. 301.

22. K. Marubashi, S. Hirano, Suzuki K.T., Toxicology Letters, 99 (1998) 43.

23. Y. Nakamura, Y. Tsumura, Y. Tonogai, T. Shibata, Y. Ito, Fund. Appl. Toxicology 37 (1997) 106.

24. D. Schubert, R. Dargusch, J. Raitano, S-W. Chan, Biochem. Biophys. Res. Comm. 342 (2006) 86.

25. J.L. Domingo, Toxicology and Environmental Health, 42 (1994) p. 123

26. D.F. Lison, Chapter 73 in Environmental and Occupational Medicine, Third Edition (W.N. Rom, Ed.), Lippincott-Raven, Philadelphia, (1998).

27. N. Lombaert, M. De Boeck, I. Decordier, E. Cundari,. D. Lison,. M. Kirsh-Volders, Toxicology Letters 154 (2004) p. 23

28. Q. Zhang, Y. Kusaka, K. Sato, J. Toxicology Envir. Health Part A 53 (1998) 423.

29. K. Jian, I. Kulaots, A. Yan, G.P. Crawford, R.H. Hurt, Carbon, 44 (2006) 2102.

30. A. Yan, X. Cheng, I. Kulaots, B.W. Sheldon, R.H. Hurt, Controlling water contact angle on carbon surfaces from 5 to 167 degrees, Carbon, in press, 2006.

31. H. Dumortier, S. Lacotte, G. Pastorin, R. Marega, W. Wu, D. Bonifazi, J. Briand, M. Prato, S. Muller, A. Bianco, Nano Letters 6 (2006) 1522-1528. 